\title{
Weight control for modelling with NURPS surfaces
}

\author{
Hendrik Speleers \\ Paul Dierckx \\ Stefan Vandewalle
}

Report TW 473, October 2006

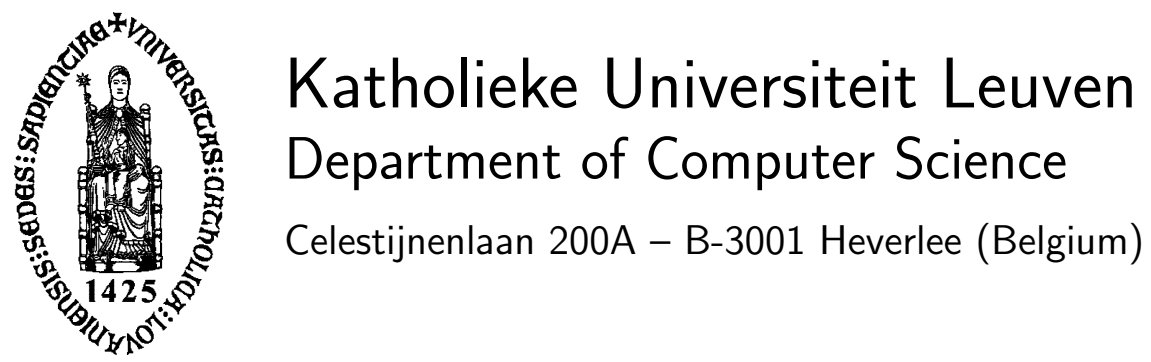




\title{
Weight control for modelling with NURPS surfaces
}

\author{
Hendrik Speleers \\ Paul Dierckx \\ Stefan Vandewalle
}

Report TW 473, October 2006

Department of Computer Science, K.U.Leuven

\begin{abstract}
NURPS surfaces are the rational extension of Powell-Sabin splines in their normalized B-spline representation. In this paper we study the influence of modifying the weights of a NURPS surface. We describe the relation between the weights associated with a control triangle and the points on the NURPS surface by means of a double volume ratio. We also extend the concept of Farin points for rational Bézier curves to NURPS surfaces, resulting in weight points and weight triangles. They admit a local weight control in a geometrically intuitive way.
\end{abstract}

Keywords : NURPS, rational splines, weight control, weight points AMS(MOS) Classification : Primary : 65D07, Secondary : 65D17, 68U07 


\title{
Weight control for modelling with NURPS surfaces
}

\author{
Hendrik Speleers, Paul Dierckx and Stefan Vandewalle \\ Department of Computer Science, Katholieke Universiteit Leuven \\ Celestijnenlaan 200A, B-3001 Leuven, Belgium
}

\begin{abstract}
NURPS surfaces are the rational extension of Powell-Sabin splines in their normalized B-spline representation. In this paper we study the influence of modifying the weights of a NURPS surface. We describe the relation between the weights associated with a control triangle and the points on the NURPS surface by means of a double volume ratio. We also extend the concept of Farin points for rational Bézier curves to NURPS surfaces, resulting in weight points and weight triangles. They admit a local weight control in a geometrically intuitive way.
\end{abstract}

Keywords: NURPS, rational splines, weight control, weight points AMS classification: $65 \mathrm{D} 07,65 \mathrm{D} 17,68 \mathrm{U} 07$

\section{Introduction}

Rational surfaces such as rational Bézier and NURBS surfaces, are commonly used in commercially available computer aided design and computer graphic packages [3]. The weights of a rational surface give the designer extra degrees of freedom compared with its non-rational counterpart. A careful selection of the weights allows one to represent patches on quadric surfaces exactly, e.g., patches on the cone and the sphere. The use of NURBS weights has also been explored for other applications, such as shape optimization and modification (see, e.g., $[4,7]$ ).

For rational Bézier curves, one can use so-called weight points (also called Farin points) as a design tool for handling the weights in a geometrically intuitive way. Given two adjacent Bézier points $\mathbf{b}_{i}$ and $\mathbf{b}_{i+1}$ with their associated weights $w_{i}$ and $w_{i+1}$, the Farin point $\mathbf{f}_{i}$ is defined as

$$
\mathbf{f}_{i}=\frac{w_{i} \mathbf{b}_{i}+w_{i+1} \mathbf{b}_{i+1}}{w_{i}+w_{i+1}}
$$

The location of $\mathbf{f}_{i}$ on the line through $\mathbf{b}_{i}$ and $\mathbf{b}_{i+1}$ determines the ratio of $w_{i}$ and $w_{i+1}$ uniquely as

$$
\frac{\left\|\mathbf{f}_{i}-\mathbf{b}_{i}\right\|}{\left\|\mathbf{f}_{i}-\mathbf{b}_{i+1}\right\|}=\frac{w_{i+1}}{w_{i}}
$$

This can be intuitively interpreted as follows: the more $\mathbf{f}_{i}$ moves towards $\mathbf{b}_{i}$, the larger $w_{i}$ is relative to $w_{i+1}$. In addition, the rational Bézier curve lies not only in the convex hull spanned by its control points $\mathbf{b}_{0}, \ldots, \mathbf{b}_{n}$, but also in the convex hull spanned by $\mathbf{b}_{0}, \mathbf{f}_{0}, \ldots, \mathbf{f}_{n-1}, \mathbf{b}_{n}[3]$. An extension of Farin points to rational Bézier surfaces is not straightforward. In [1, 9] some generalizations are proposed.

In a similar way as classical B-spline surfaces are generalized to NURBS surfaces, Windmolders and Dierckx [11] developed the rational extension of Powell-Sabin splines, so-called NURPS surfaces. These $C^{1}$-continuous spline surfaces can be represented in a compact B-spline basis with an intuitive geometrical interpretation involving tangent control triangles. This paper focuses on the 


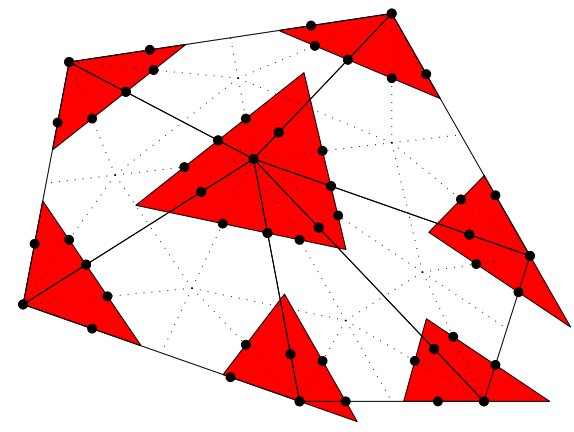

(a) PS refinement with PS triangles

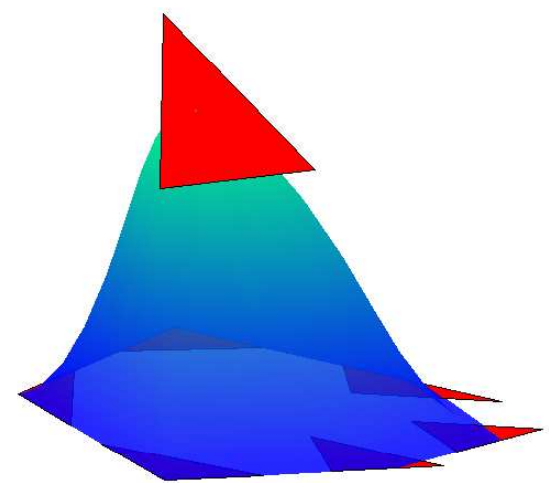

(b) NURPS surface with control triangles

Figure 1: (a) A PS refinement $\Delta^{*}$ (dotted lines) of a given triangulation $\Delta$ (solid lines) with a set of suitable PS triangles. (b) A NURPS surface on $\Delta^{*}$ with corresponding control triangles.

use of NURPS weights for shape modelling. We show that the weights of NURPS surfaces can be interpreted geometrically, resulting in an intuitive way of surface manipulation. We define weight points and weight triangles for NURPS, analogous to the Farin points (1.1) for rational Bézier curves.

\section{$2 \quad$ NURPS surfaces}

Consider a simply connected subset $\Omega \subset \mathbb{R}^{2}$ with polygonal boundary $\partial \Omega$. Assume a conforming triangulation $\Delta$ of $\Omega$ is given, consisting of $n$ vertices $V_{i}, i=1, \ldots, n$. The Powell-Sabin (PS) refinement $\Delta^{*}$ is a partition of $\Delta$, obtained by splitting each triangle $\rho_{j}$ into six smaller triangles with a common vertex [6]. In Figure 1(a) such a PS refinement of a given triangulation is drawn in dotted lines.

A Powell-Sabin spline is a piecewise quadratic polynomial with global $C^{1}$-continuity defined on $\Delta^{*}$. Dierckx [2] presented how to construct a normalized B-spline basis for PS splines. With each vertex $V_{i}$, one can associate a so-called PS triangle $t_{i}\left(Q_{i, 1}, Q_{i, 2}, Q_{i, 3}\right)$. There exists a unique relation between the PS triangles and the PS B-splines $B_{i}^{j}(u, v)$. If the PS triangles contain certain points, the so-called PS points, the PS B-splines form a convex partition of unity. The vertex itself is a PS point, together with the midpoints of all edges in the PS refinement $\Delta^{*}$ containing $V_{i}$.

Definition 2.1. A non-uniform rational Powell-Sabin (NURPS) surface $\mathbf{s}(u, v)$ is defined as

$$
\mathbf{s}(u, v)=\sum_{i=1}^{n} \sum_{j=1}^{3} \mathbf{c}_{i, j} \phi_{i}^{j}(u, v), \quad \text { with } \quad \phi_{i}^{j}(u, v)=\frac{w_{i, j} B_{i}^{j}(u, v)}{\sum_{i=1}^{n} \sum_{j=1}^{3} w_{i, j} B_{i}^{j}(u, v)}, \quad(u, v) \in \Omega,
$$

where $\mathbf{c}_{i, j}=\left(c_{i, j}^{x}, c_{i, j}^{y}, c_{i, j}^{z}\right)$ are the NURPS control points, $B_{i}^{j}(u, v)$ are the normalized PS B-splines, and $w_{i, j}$ are positive weights.

Windmolders and Dierckx [11] showed how NURPS surfaces can be represented in a BernsteinBézier formulation by means of rational Bézier ordinates. Using the rational de Casteljau algorithm, the NURPS surfaces can then be evaluated and manipulated in a stable way. With each vertex $V_{i}$, one can define a control triangle $T_{i}\left(\mathbf{c}_{i, 1}, \mathbf{c}_{i, 2}, \mathbf{c}_{i, 3}\right)$. This triangle is tangent to the surface at the 
point $\mathbf{s}\left(V_{i}\right)=\hat{\alpha}_{i, 1} \mathbf{c}_{i, 1}+\hat{\alpha}_{i, 2} \mathbf{c}_{i, 2}+\hat{\alpha}_{i, 3} \mathbf{c}_{i, 3}$, where

$$
\hat{\alpha}_{i, j}=\frac{w_{i, j} \alpha_{i, j}}{\sum_{k=1}^{3} w_{i, k} \alpha_{i, k}},
$$

and $\left(\alpha_{i, 1}, \alpha_{i, 2}, \alpha_{i, 3}\right)$ are the barycentric coordinates of vertex $V_{i}$ with respect to PS triangle $t_{i}$. With particular choices for the control points the NURPS can exactly represent patches on quadric surfaces, and degeneration of the control triangles allows the modelling of certain special effects such as cups and corners [12]. Recently also local subdivision has been considered [8]. Figure 1(b) shows a NURPS surface with corresponding control triangles.

\section{Weight modification}

We first consider the influence of modifying a single weight on the NURPS surface. Increasing the weight $w_{i, j}$ results in a local attraction of the surface towards the control point $\mathbf{c}_{i, j}$. The theorem below is valid for any NURBS-like rational surface (see [5] for a proof with respect to NURBS surfaces), and in particular also for NURPS surfaces.

Theorem 3.1. Let $(\bar{u}, \bar{v})$ be a fixed domain point within the molecule of $V_{i}$, and define the points $\mathbf{q}_{i, j}=\mathbf{s}\left(\bar{u}, \bar{v} ; w_{i, j}=1\right)$ and $\mathbf{r}_{i, j}=\mathbf{s}\left(\bar{u}, \bar{v} ; w_{i, j}=0\right)$. The surface point $\mathbf{p}_{i, j}=\mathbf{s}\left(\bar{u}, \bar{v} ; w_{i, j}\right)$ can be written as a convex combination of $\mathbf{r}_{i, j}$ and $\mathbf{c}_{i, j}$, i.e.,

$$
\mathbf{p}_{i, j}=\left(1-\phi_{i}^{j}(\bar{u}, \bar{v})\right) \mathbf{r}_{i, j}+\phi_{i}^{j}(\bar{u}, \bar{v}) \mathbf{c}_{i, j},
$$

and the weight $w_{i, j}$ can be geometrically related to $\mathbf{p}_{i, j}$ by

$$
w_{i, j}=\frac{\left\|\mathbf{r}_{i, j}-\mathbf{p}_{i, j}\right\|\left\|\mathbf{c}_{i, j}-\mathbf{q}_{i, j}\right\|}{\left\|\mathbf{r}_{i, j}-\mathbf{q}_{i, j}\right\|\left\|\mathbf{c}_{i, j}-\mathbf{p}_{i, j}\right\|} .
$$

Using relation (3.2), the corresponding value of $w_{i, j}$ can be easily determined when one moves the surface point $\mathbf{p}_{i, j}$ from $\mathbf{r}_{i, j}\left(w_{i, j}=0\right)$ to $\mathbf{c}_{i, j}\left(w_{i, j}=\infty\right)$ along a straight line. It is illustrated in Figure 2(a). We now study the effect of changing simultaneously the three weights associated with a control triangle.

Lemma 3.1. The surface point $\mathbf{p}_{i}=\mathbf{s}\left(\bar{u}, \bar{v} ; w_{i, 1}, w_{i, 2}, w_{i, 3}\right)$ can be written as a convex combination of the point $\mathbf{r}_{i}=\mathbf{s}\left(\bar{u}, \bar{v} ; w_{i, 1}=w_{i, 2}=w_{i, 3}=0\right)$ and the three control points $\mathbf{c}_{i, j}, j=1,2,3$ :

$$
\mathbf{p}_{i}=\left(1-\phi_{i}^{1}(\bar{u}, \bar{v})-\phi_{i}^{2}(\bar{u}, \bar{v})-\phi_{i}^{3}(\bar{u}, \bar{v})\right) \mathbf{r}_{i}+\phi_{i}^{1}(\bar{u}, \bar{v}) \mathbf{c}_{i, 1}+\phi_{i}^{2}(\bar{u}, \bar{v}) \mathbf{c}_{i, 2}+\phi_{i}^{3}(\bar{u}, \bar{v}) \mathbf{c}_{i, 3} .
$$

Proof. If we vary the three weights $w_{i, j}$ one by one, relation (3.1) can be applied to find that

$$
\begin{aligned}
\mathbf{p}_{i} & =\left(1-\phi_{i}^{1}(\bar{u}, \bar{v})\right) \mathbf{r}_{i, 1}+\phi_{i}^{1}(\bar{u}, \bar{v}) \mathbf{c}_{i, 1} \\
\mathbf{r}_{i, 1} & =\left(1-\phi_{i}^{2}\left(\bar{u}, \bar{v} ; w_{i, 1}=0\right)\right) \mathbf{r}_{i, 12}+\phi_{i}^{2}\left(\bar{u}, \bar{v} ; w_{i, 1}=0\right) \mathbf{c}_{i, 2}, \\
\mathbf{r}_{i, 12} & =\left(1-\phi_{i}^{3}\left(\bar{u}, \bar{v} ; w_{i, 1}=w_{i, 2}=0\right)\right) \mathbf{r}_{i}+\phi_{i}^{3}\left(\bar{u}, \bar{v} ; w_{i, 1}=w_{i, 2}=0\right) \mathbf{c}_{i, 3} .
\end{aligned}
$$

Hence,

$$
\begin{aligned}
\mathbf{p}_{i}=(1 & \left.-\phi_{i}^{1}(\bar{u}, \bar{v})\right)\left(1-\phi_{i}^{2}\left(\bar{u}, \bar{v} ; w_{i, 1}=0\right)\right)\left(1-\phi_{i}^{3}\left(\bar{u}, \bar{v} ; w_{i, 1}=w_{i, 2}=0\right)\right) \mathbf{r}_{i} \\
& +\phi_{i}^{1}(\bar{u}, \bar{v}) \mathbf{c}_{i, 1}+\left(1-\phi_{i}^{1}(\bar{u}, \bar{v})\right) \phi_{i}^{2}\left(\bar{u}, \bar{v} ; w_{i, 1}=0\right) \mathbf{c}_{i, 2} \\
& +\left(1-\phi_{i}^{1}(\bar{u}, \bar{v})\right)\left(1-\phi_{i}^{2}\left(\bar{u}, \bar{v} ; w_{i, 1}=0\right)\right) \phi_{i}^{3}\left(\bar{u}, \bar{v} ; w_{i, 1}=w_{i, 2}=0\right) \mathbf{c}_{i, 3} .
\end{aligned}
$$

Since

$$
\begin{aligned}
\phi_{i}^{2}(\bar{u}, \bar{v}) & =\left(1-\phi_{i}^{1}(\bar{u}, \bar{v})\right) \phi_{i}^{2}\left(\bar{u}, \bar{v} ; w_{i, 1}=0\right), \\
\phi_{i}^{3}(\bar{u}, \bar{v}) & =\left(1-\phi_{i}^{1}(\bar{u}, \bar{v})\right)\left(1-\phi_{i}^{2}\left(\bar{u}, \bar{v} ; w_{i, 1}=0\right)\right) \phi_{i}^{3}\left(\bar{u}, \bar{v} ; w_{i, 1}=w_{i, 2}=0\right),
\end{aligned}
$$

we obtain relation (3.3). 


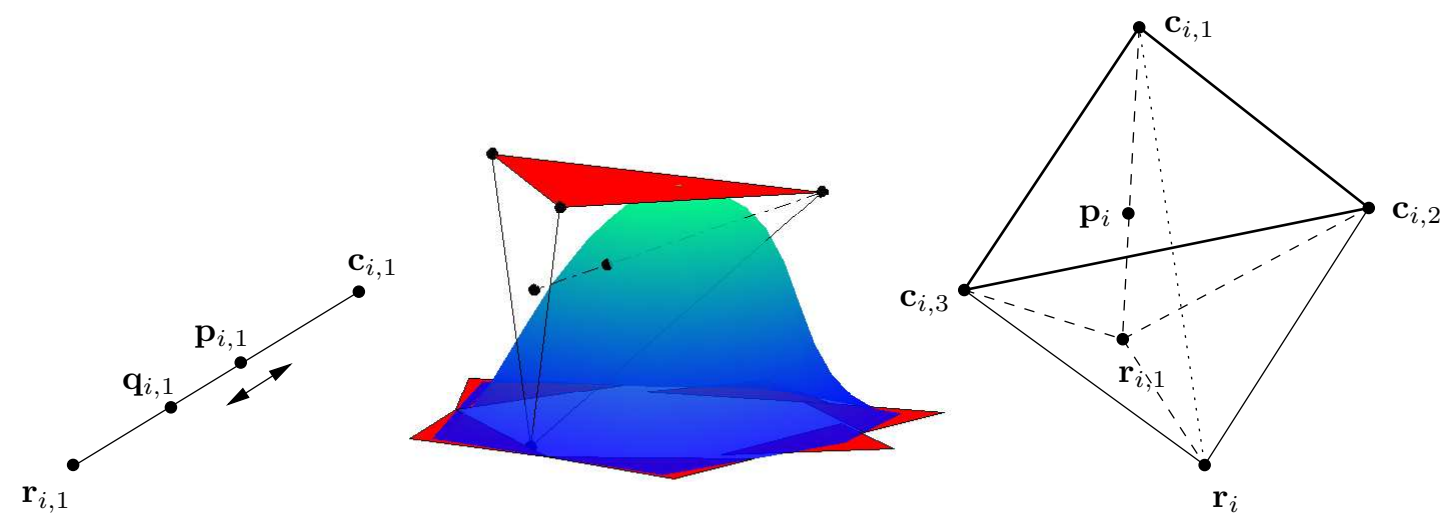

(a) Modifying one weight

(b) Modifying three weights

Figure 2: (a) Modifying the weight $w_{i, j}$ will move the surface point $\mathbf{p}_{i, 1}$ along the line $\mathbf{c}_{i, 1}-\mathbf{r}_{i, 1}$. (b) Modifying the weights associated with control triangle $T_{i}$ will vary the surface point $\mathbf{p}_{i}$ within the tetrahedron spanned by $\mathbf{c}_{i, 1}, \mathbf{c}_{i, 2}, \mathbf{c}_{i, 3}$ and $\mathbf{r}_{i}$. The point $\mathbf{r}_{i, 1}$ is located on the triangle formed by $\mathbf{c}_{i, 2}, \mathbf{c}_{i, 3}, \mathbf{r}_{i}$.

The lemma implies that the position of the surface point $\mathbf{p}_{i}$ will stay within the tetrahedron spanned by the points $\mathbf{c}_{i, 1}, \mathbf{c}_{i, 2}, \mathbf{c}_{i, 3}$ and $\mathbf{r}_{i}$, when the values of the three weights $w_{i, j}$ are modified. This is illustrated in Figure 2(b).

Remark 3.1. The point $\mathbf{r}_{i, 1}$, defined in Theorem 3.1, is the intersection point between the line $\mathbf{p}_{i}-\mathbf{c}_{i, 1}$ and the triangle formed by $\mathbf{c}_{i, 2}, \mathbf{c}_{i, 3}, \mathbf{r}_{i}$. It satisfies

$$
\mathbf{r}_{i, 1}=\left(1-\phi_{i}^{2 *}-\phi_{i}^{3 *}\right) \mathbf{r}_{i}+\phi_{i}^{2 *} \mathbf{c}_{i, 2}+\phi_{i}^{3 *} \mathbf{c}_{i, 3}
$$

with $\phi_{i}^{2 *}=\phi_{i}^{2}(\bar{u}, \bar{v}) /\left(1-\phi_{i}^{1}(\bar{u}, \bar{v})\right)$ and $\phi_{i}^{3 *}=\phi_{i}^{3}(\bar{u}, \bar{v}) /\left(1-\phi_{i}^{1}(\bar{u}, \bar{v})\right)$. Similar expressions are valid for $\mathbf{r}_{i, 2}$ and $\mathbf{r}_{i, 3}$.

Theorem 3.2. Denote $\mathcal{V}(\cdot, \cdot, \cdot, \cdot)$ as the volume of the tetrahedron spanned by the four given points, and define $j^{\prime}$ and $j^{\prime \prime}$ as $j^{\prime}=1+(j \bmod 3)$ and $j^{\prime \prime}=1+\left(j^{\prime} \bmod 3\right)$ respectively. Given a domain point $(\bar{u}, \bar{v})$ within the molecule of $V_{i}$, the three weights $w_{i, j}$ associated with the control triangle $T_{i}\left(\mathbf{c}_{i, 1}, \mathbf{c}_{i, 2}, \mathbf{c}_{i, 3}\right)$ can be geometrically related to the surface point $\mathbf{p}_{i}=\mathbf{s}\left(\bar{u}, \bar{v} ; w_{i, 1}, w_{i, 2}, w_{i, 3}\right)$ as

$$
w_{i, j}=\frac{\mathcal{V}\left(\mathbf{p}_{i}, \mathbf{r}_{i}, \mathbf{c}_{i, j^{\prime}}, \mathbf{c}_{i, j^{\prime \prime}}\right) \mathcal{V}\left(\mathbf{q}_{i}, \mathbf{c}_{i, 1}, \mathbf{c}_{i, 2}, \mathbf{c}_{i, 3}\right)}{\mathcal{V}\left(\mathbf{q}_{i}, \mathbf{r}_{i}, \mathbf{c}_{i, j^{\prime}}, \mathbf{c}_{i, j^{\prime \prime}}\right) \mathcal{V}\left(\mathbf{p}_{i}, \mathbf{c}_{i, 1}, \mathbf{c}_{i, 2}, \mathbf{c}_{i, 3}\right)}
$$

where $\mathbf{q}_{i}=\mathbf{s}\left(\bar{u}, \bar{v} ; w_{i, 1}=w_{i, 2}=w_{i, 3}=1\right)$ and $\mathbf{r}_{i}=\mathbf{s}\left(\bar{u}, \bar{v} ; w_{i, 1}=w_{i, 2}=w_{i, 3}=0\right)$.

Proof. Let $\psi_{i}^{j}(\bar{u}, \bar{v})=\phi_{i}^{j}\left(\bar{u}, \bar{v} ; w_{i, 1}=w_{i, 2}=w_{i, 3}=1\right)$, then by Lemma 3.1 we can write

$$
\mathbf{q}_{i}=\left(1-\psi_{i}^{1}(\bar{u}, \bar{v})-\psi_{i}^{2}(\bar{u}, \bar{v})-\psi_{i}^{3}(\bar{u}, \bar{v})\right) \mathbf{r}_{i}+\psi_{i}^{1}(\bar{u}, \bar{v}) \mathbf{c}_{i, 1}+\psi_{i}^{2}(\bar{u}, \bar{v}) \mathbf{c}_{i, 2}+\psi_{i}^{3}(\bar{u}, \bar{v}) \mathbf{c}_{i, 3} .
$$

Using expression (2.1) for $\phi_{i}^{j}(\bar{u}, \bar{v})$ and $\psi_{i}^{j}(\bar{u}, \bar{v})$, we have

$$
\frac{\phi_{i}^{j}(\bar{u}, \bar{v})}{\psi_{i}^{j}(\bar{u}, \bar{v})}=\frac{w_{i, j}\left(\sum_{l} B_{i}^{l}(\bar{u}, \bar{v})+\sum_{k \neq i} \sum_{l} w_{k, l} B_{k}^{l}(\bar{u}, \bar{v})\right)}{\sum_{k} \sum_{l} w_{k, l} B_{k}^{l}(\bar{u}, \bar{v})}=w_{i, j} \frac{1-\phi_{i}^{1}(\bar{u}, \bar{v})-\phi_{i}^{2}(\bar{u}, \bar{v})-\phi_{i}^{3}(\bar{u}, \bar{v})}{1-\psi_{i}^{1}(\bar{u}, \bar{v})-\psi_{i}^{2}(\bar{u}, \bar{v})-\psi_{i}^{3}(\bar{u}, \bar{v})} .
$$

Combining this relation with the convex combinations (3.3) and (3.6) of $\mathbf{p}_{i}$ and $\mathbf{q}_{i}$, we obtain equation (3.5). 


\section{Weight points and weight triangles}

One can use the concept of the above mentioned tetrahedra (see Figure 2) to determine the weights in a geometrical way. The designer selects an arbitrary surface point $\mathbf{p}_{i}$ and a control triangle. When he/she moves that point within the corresponding tetrahedron, a new NURPS surface with the same control triangles can be found that passes through the new position of $\mathbf{p}_{i}$ by calculating the values of the weights via formula (3.5).

Yet, when the designer picks the tangent point of control triangle $T_{i}\left(\mathbf{c}_{i, 1}, \mathbf{c}_{i, 2}, \mathbf{c}_{i, 3}\right)$ to the surface, i.e. $\mathbf{p}_{i}=\mathbf{s}\left(V_{i}\right)$, formula (3.5) is not usable anymore since both numerator and denominator are zero. From [11] we know that in this case

$$
\mathbf{p}_{i}=\hat{\alpha}_{i, 1} \mathbf{c}_{i, 1}+\hat{\alpha}_{i, 2} \mathbf{c}_{i, 2}+\hat{\alpha}_{i, 3} \mathbf{c}_{i, 3}
$$

with $\hat{\alpha}_{i, j}$ defined as in $(2.2)$, and

$$
\mathbf{q}_{i}=\alpha_{i, 1} \mathbf{c}_{i, 1}+\alpha_{i, 2} \mathbf{c}_{i, 2}+\alpha_{i, 3} \mathbf{c}_{i, 3}
$$

Let $\mathcal{A}(\cdot, \cdot, \cdot)$ be the area of the triangle spanned by three given points. From $(2.2),(4.1),(4.2)$ and $\hat{\alpha}_{i, 1}+\hat{\alpha}_{i, 2}+\hat{\alpha}_{i, 3}=1$, we can write the weights $w_{i, j}$ as

$$
w_{i, j}=K \frac{\hat{\alpha}_{i, j}}{\alpha_{i, j}}=K \frac{\mathcal{A}\left(\mathbf{p}_{i}, \mathbf{c}_{i, j^{\prime}}, \mathbf{c}_{i, j^{\prime \prime}}\right)}{\mathcal{A}\left(\mathbf{q}_{i}, \mathbf{c}_{i, j^{\prime}}, \mathbf{c}_{i, j^{\prime \prime}}\right)}
$$

for an arbitrary value $K>0$, and with $j^{\prime}$ and $j^{\prime \prime}$ defined as in Theorem 3.2.

Equations (4.1) and (4.3) allow us to define a weight point corresponding to each vertex $V_{i}$. Such a point is characterized by its position $\mathbf{s}\left(V_{i}\right)$ and by a scaling factor $K$. The three weights $w_{i, j}$ are then uniquely defined by its barycentric coordinates $\left(\hat{\alpha}_{i, 1}, \hat{\alpha}_{i, 2}, \hat{\alpha}_{i, 3}\right)$ with respect to $T_{i}\left(\mathbf{c}_{i, 1}, \mathbf{c}_{i, 2}, \mathbf{c}_{i, 3}\right)$ up to the factor $K$ using (4.3). A designer can freely move the weight point within the control triangle $T_{i}$. Since its position is the tangent point of $T_{i}$ to the NURPS surface, the effect of the movement will be intuitive to the designer. This is illustrated in the first two pictures in Figure 3 . One can use the scaling factor $K$ to determine the relative importance of the considered three weights with respect to the other weights. Note that $K$ can be interpreted as a weighted mean of these weights, i.e., $K=\alpha_{i, 1} w_{i, 1}+\alpha_{i, 2} w_{i, 2}+\alpha_{i, 3} w_{i, 3}$. The larger the value of $K$, the more the NURPS surface will be attracted to the control triangle. A cusp can be simulated by reducing the scaling factor. The effect of changing the scaling factor is illustrated in the bottom two pictures of Figure 3. Similar effects can be obtained by rescaling (enlarging/reducing) the control triangles. However, changing $K$ has the advantage that the designer can still work with the same control triangles.

Based on the concept of a weight point, we can derive a relation similar to (1.2) for Farin points. To that end, we consider Remark 3.1 and apply the result for the case $\mathbf{p}_{i}$ is the weight point. The point $\mathbf{r}_{i, j}$ is the intersection point between the line $\mathbf{p}_{i}-\mathbf{c}_{i, j}$ and the edge of $T_{i}$ opposite to $\mathbf{c}_{i, j}$, see Figure 4. It can be written as

$$
\mathbf{r}_{i, j}=\frac{\hat{\alpha}_{i, j^{\prime}} \mathbf{c}_{i, j^{\prime}}+\hat{\alpha}_{i, j^{\prime \prime}} \mathbf{c}_{i, j^{\prime \prime}}}{\hat{\alpha}_{i, j^{\prime}}+\hat{\alpha}_{i, j^{\prime \prime}}} .
$$

We define the weight triangle $w T_{i}$ as the triangle formed by the three points $\mathbf{r}_{i, j}$. Combining (4.3) with (4.4), we obtain the next property.

Property 4.1. The weight triangle $w T_{i}\left(\mathbf{r}_{i, 1}, \mathbf{r}_{i, 2}, \mathbf{r}_{i, 3}\right)$ is related to the three weights $w_{i, j}$ by

$$
\frac{\alpha_{i, j^{\prime}}\left\|\mathbf{r}_{i, j}-\mathbf{c}_{i, j^{\prime}}\right\|}{\alpha_{i, j^{\prime \prime}}\left\|\mathbf{r}_{i, j}-\mathbf{c}_{i, j^{\prime \prime}}\right\|}=\frac{w_{i, j^{\prime \prime}}}{w_{i, j^{\prime}}}
$$




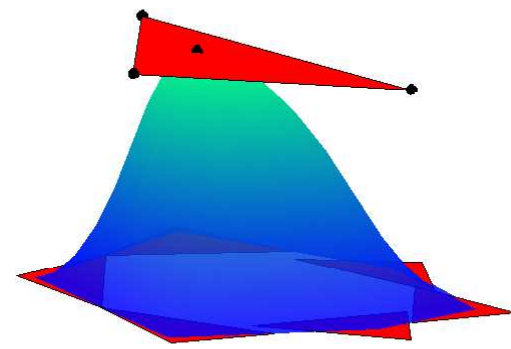

(a) Original NURPS

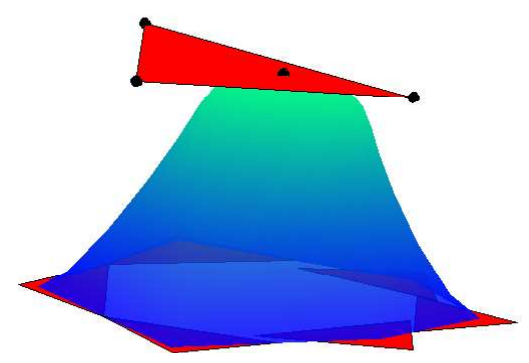

(c) Enlarging scaling factor

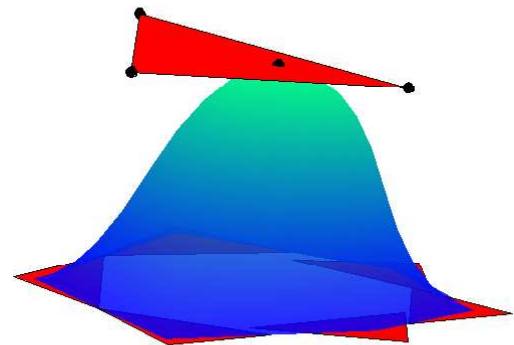

(b) Moving weight (tangent) point

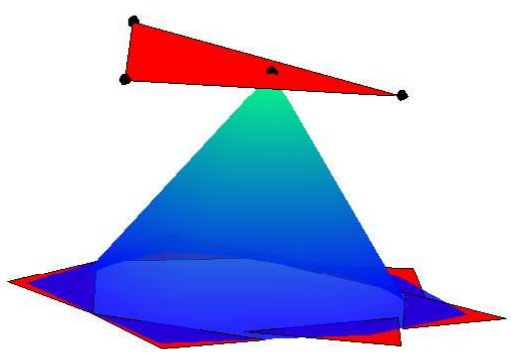

(d) Reducing scaling factor

Figure 3: The effect of moving the weight point $\mathbf{p}_{i}$ inside the control triangle and modifying the scaling factor $K$. The considered weight point and control points are indicated with bullets.

Remark 4.1. If we use a uniform triangulation (see, e.g., [10]), then $\alpha_{i, 1}=\alpha_{i, 2}=\alpha_{i, 3}=1 / 3$, and (4.5) simplifies to

$$
\frac{\left\|\mathbf{r}_{i, j}-\mathbf{c}_{i, j^{\prime}}\right\|}{\left\|\mathbf{r}_{i, j}-\mathbf{c}_{i, j^{\prime \prime}}\right\|}=\frac{w_{i, j^{\prime \prime}}}{w_{i, j^{\prime}}} .
$$

which is identical to relation (1.2) for Farin points.

The following two properties can be shown using (4.1), (4.4) and some elementary calculations.

Property 4.2. The weight triangle $w T_{i}$ is tangent to the surface at the weight point $\mathbf{p}_{i}$, where

$$
\mathbf{p}_{i}=\frac{1-\hat{\alpha}_{i, 1}}{2} \mathbf{r}_{i, 1}+\frac{1-\hat{\alpha}_{i, 2}}{2} \mathbf{r}_{i, 2}+\frac{1-\hat{\alpha}_{i, 3}}{2} \mathbf{r}_{i, 3},
$$

with $\hat{\alpha}_{i, j}$ defined in (2.2).

Property 4.3. The areas of weight triangle $w T_{i}$ and control triangle $T_{i}$ are related as

$$
\mathcal{A}\left(\mathbf{r}_{i, 1}, \mathbf{r}_{i, 2}, \mathbf{r}_{i, 3}\right)=\frac{2 \hat{\alpha}_{i, 1} \hat{\alpha}_{i, 2} \hat{\alpha}_{i, 3}}{\left(1-\hat{\alpha}_{i, 1}\right)\left(1-\hat{\alpha}_{i, 2}\right)\left(1-\hat{\alpha}_{i, 3}\right)} \mathcal{A}\left(\mathbf{c}_{i, 1}, \mathbf{c}_{i, 2}, \mathbf{c}_{i, 3}\right) .
$$

The next property is similar to the convex hull property of Farin points for rational Bézier curves.

Property 4.4. Associate with each vertex $V_{i}$ a triangle $w t_{i}$ defined by

$$
w t_{i}\left(\frac{\alpha_{i, 2} Q_{i, 2}+\alpha_{i, 3} Q_{i, 3}}{\alpha_{i, 2}+\alpha_{i, 3}}, \frac{\alpha_{i, 3} Q_{i, 3}+\alpha_{i, 1} Q_{i, 1}}{\alpha_{i, 3}+\alpha_{i, 1}}, \frac{\alpha_{i, 1} Q_{i, 1}+\alpha_{i, 2} Q_{i, 2}}{\alpha_{i, 1}+\alpha_{i, 2}}\right) .
$$

The NURPS surface lies inside the convex hull of the weight triangles, if each wt $t_{i}$ contains all PS points associated with $V_{i}$. 

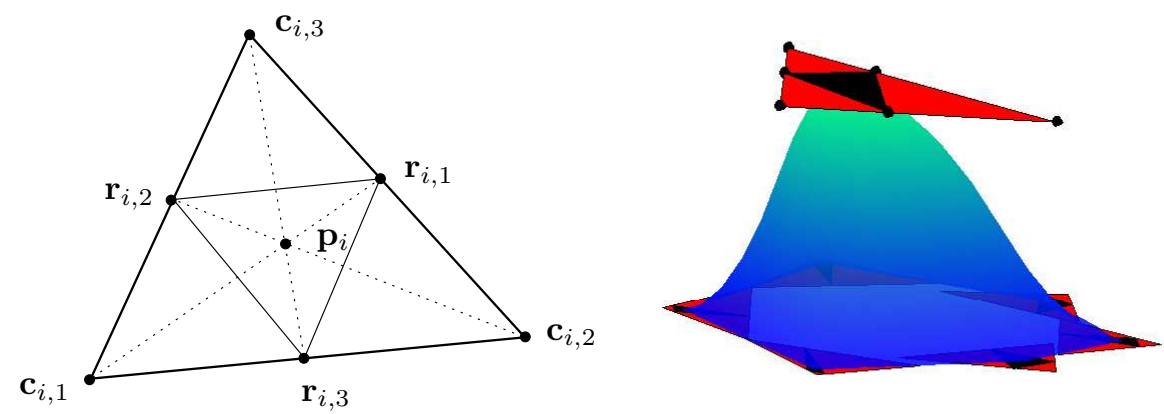

Figure 4: The weight point $\mathbf{p}_{i}$ in control triangle $T_{i}\left(\mathbf{c}_{i, 1}, \mathbf{c}_{i, 2}, \mathbf{c}_{i, 3}\right)$ uniquely defines the weight triangle $w T_{i}\left(\mathbf{r}_{i, 1}, \mathbf{r}_{i, 2}, \mathbf{r}_{i, 3}\right)$.

Proof. If we consider the B-splines $\tilde{B}_{i}^{j}(u, v)$ corresponding to the triangles $w t_{i}$ as PS triangles, it can be checked that the given NURPS surface has a corresponding representation with the points $\mathbf{r}_{i, j}$ as control points and weights $\tilde{w}_{i, j}=\left(\alpha_{i, j^{\prime}} w_{i, j^{\prime}}+\alpha_{i, j^{\prime \prime}} w_{i, j^{\prime \prime}}\right) /\left(\alpha_{i, j^{\prime}}+\alpha_{i, j^{\prime \prime}}\right)$. Thus, if all the triangles $w t_{i}$ contain the corresponding PS points, the B-splines $\tilde{B}_{i}^{j}(u, v)$ will form a convex partition of unity [2].

Remark 4.2. The condition in Property 4.4 is independent of the choice of weights. It should be noted that this condition is not always satisfied in practice. In [2], e.g., PS triangles $t_{i}$ of minimal area are computed. Since the triangles $w t_{i}$ are necessarily smaller than $t_{i}$, the former cannot in this case contain all PS points.

Since the weight triangles bear a strong resemblance to Farin points, one can use them to determine the values of the weights geometrically. The designer can move the corners of the weight triangle along the edges of the control triangle, and the corresponding weights are then calculated using (4.5). However, only two of the three corners are allowed to be chosen independently. Therefore, the use of the proposed weight points may be more appropriate.

\section{Concluding remarks}

In this paper we investigated the influence of the weights on a NURPS surface, the rational extension of Powell-Sabin splines. We derived the relation between the weights associated with a control triangle and the points on the NURPS surface. It can be geometrically interpreted as a double volume ratio.

We have defined weight points for controlling the weights of a NURPS surface in an intuitive way. A designer can freely move these points within the corresponding control triangles. The weight points have the nice property that they are the tangent points of the control triangles to the NURPS surface. We also defined weight triangles, which admit similar properties as the Farin points for a rational Bézier curve.

\section{Acknowledgement}

Hendrik Speleers is a Research Assistant of the Fund for Scientific Research Flanders (Belgium). 


\section{References}

[1] G. Albrecht. A note on Farin points for rational triangular Bézier patches. Comput. Aided Geom. Design, 12:507-512, 1995.

[2] P. Dierckx. On calculating normalized Powell-Sabin B-splines. Comput. Aided Geom. Design, 15(1):61-78, 1997.

[3] G. Farin. NURBS: from projective geometry to practical use. A.K. Peters, 2nd edition, 1999.

[4] W. Hohenberger and T. Reuding. Smoothing rational B-spline curves using the weights in an optimization procedure. Comput. Aided Geom. Design, 12:837-848, 1995.

[5] L. Piegl and W. Tiller. The NURBS book. Springer Verlag, New York, 2nd edition, 1996.

[6] M.J.D. Powell and M.A. Sabin. Piecewise quadratic approximations on triangles. ACM Trans. Math. Softw., 3:316-325, 1977.

[7] J. Sanchez-Reyes. A simple technique for NURBS shape modification. IEEE Comput. Graphics Appl., 17(1):52-59, 1997.

[8] H. Speleers, P. Dierckx, and S. Vandewalle. Local subdivision of Powell-Sabin splines. Comput. Aided Geom. Design, 23(5):446-462, 2006.

[9] H. Theisel. Using Farin points for rational Bézier surfaces. Comput. Aided Geom. Design, 16:817-835, 1999.

[10] J. Windmolders and P. Dierckx. Subdivision of uniform Powell-Sabin splines. Comput. Aided Geom. Design, 16:301-315, 1999.

[11] J. Windmolders and P. Dierckx. From PS-splines to NURPS. In A. Cohen, C. Rabut, and L.L. Schumaker, editors, Proceedings of Curve and Surface Fitting, Saint-Malo 1999, pages 45-54. Vanderbilt University Press, 2000.

[12] J. Windmolders and P. Dierckx. NURPS for special effects and quadrics. In T. Lyche and L.L. Schumaker, editors, Proceedings of Mathematical Methods for Curves and Surfaces, Oslo 2000, pages 527 - 534. Vanderbilt University Press, 2001. 